\title{
REPRODUCING KERNELS AND PRINCIPAL FUNCTIONS ${ }^{1}$
}

\section{BURTON RODIN ${ }^{2}$}

1. Introduction. Using the notation and terminology of AhlforsSario [3], we shall denote by $\Gamma_{h}$ the Hilbert space of square integrable harmonic differentials on a Riemann surface $W$. Let $\gamma$ be a 1-chain on $W$ and let $\psi_{\gamma}$ be the element in $\Gamma_{h}$ with the property, $\left(\omega, \psi_{\gamma}\right)$ $=\int_{\gamma} \omega$ for all $\omega \in \Gamma_{h}$. We refer to $\psi_{\gamma}$ as a reproducing kernel for periods for $\Gamma_{h}$. Let $\zeta$ be a point of $W$ and $z=x+i y$ be a local parameter near $\zeta$ such that $z(\zeta)=0$. A $\Gamma_{h}$-kernel for $n$th derivatives at $\zeta$ is a differential $\psi_{\zeta} \in \Gamma_{h}$ which satisfies $\left(\omega, \psi_{\zeta}\right)=\left(\partial^{n} / \partial x^{n}\right) u(0)$ for all $\omega \in \Gamma_{h}$ where $\omega=d u(z)$ near $\zeta$. This kernel is uniquely determined by $\zeta$, the uniformizer $z$, and the positive integer $n$. If in the above definitions we replace $\Gamma_{h}$ by one of its subspaces then we shall refer to the corresponding kernels as reproducing kernels for that subspace. It is easily seen that such a kernel is the orthogonal projection of a $\Gamma_{h^{-}}$ kernel.

The existence of these kernels is well known (see $[1 ; 3])$. The possibility of expressing them explicitly in terms of principal harmonic functions (the existence of which has been proved constructively by Sario [5]) was first investigated by Weill [6]. In this paper the kernels for the spaces $\Gamma_{h}, \Gamma_{h s e}, \Gamma_{h e}, \Gamma_{h m}, \Gamma_{h 0}, \Gamma_{h e} \cap \Gamma_{h s e}^{*}, \Gamma_{h 0} \cap \Gamma_{h s e}^{*}, \Gamma_{a}, \Gamma_{a s e}$, and some spaces associated with a regular partition of the ideal boundary of the surface are found in terms of principal functions.

$\S \S 9$ and 10 deal with some applications of these results. A characterization of Ahlfors' class of quasi-rational functions is given in terms of principal functions. A Riemann-Roch type theorem is proved for arbitrary Riemann surfaces which reduces to a theorem of Royden [4] in case the surface is of class $O_{K D}$.

2. Reproducing kernels. We shall apply the general method of normal operators [3] to the following situation. Let $h$ be a harmonic function defined in a boundary neighborhood of a Riemann surface $W^{\prime}$ and assume that $h$ has zero flux along the ideal boundary of $W^{\prime}$. The principal operator $L_{0}$ maps $h$ into a function $p_{0 h}$ which is harmonic on $W^{\prime}$ and is characterized up to an additive constant by the

Received by the editors October 24, 1961.

${ }^{1}$ Work supported by the N.S.F., the O.O.R. contract number DA-04-495-ORD722, and the A.F.O.S.R. contract number AF49(638)-574.

2 Parts of this paper are contained in a doctoral dissertation submitted to the University of California, Los Angeles. The author is indebted to Professor Leo Sario for his helpful guidance. 
property that the normal derivative of $p_{0 h}-h$ vanishes identically along the ideal boundary of $W^{\prime}$. When $W^{\prime}$ is the complement of a finite subset $\left\{\zeta_{1}, \cdots, \zeta_{n}\right\}$ of a Riemann surface $W$ then $p_{0 h}-h$ has a removable singularity at each $\zeta_{i}$. We say that it has $L_{0}$ behavior at the ideal boundary of $W$. Let $P$ be a regular partition of this ideal boundary $\left[3\right.$, p. 165]. The $(P) L_{1}$ principal operator associates to $h$ a function $p_{P h}$, harmonic on $W^{\prime}$ with the singularity $h$ at each $\zeta_{i}$. Furthermore, $p_{P h}-h$ is constant on each ideal boundary component of $W$ determined by $P$ and has zero flux over each $P$-dividing cycle.

Let $I$ and $Q$ denote, respectively, the identity and the canonical partitions of the ideal boundary of $W$ in the sense of [3]. In terms of a uniformizer $z$ near $\zeta \in W$ such that $z(\zeta)=0$, we let $s=\operatorname{Re} z^{-n}$ and $t=\operatorname{Im} z^{-n}$ for a positive integer $n$, and define $s \equiv t \equiv 0$ outside of a compact subset of $W$. The differential $d p_{I_{s}}+d p_{I t}^{*}$ has a removable singularity at $\zeta$ and is an element of $\Gamma_{h}$.

TheOREM 1. The differential $d p_{I_{s}}+d p_{I t}^{*}$ reproduces for the space $\Gamma_{h}$. Explicitly, we have

$$
\left(\omega, d p_{I_{s}}+d p_{I t}^{*}\right)=-\frac{2 \pi}{(n-1) !} \frac{\partial^{n}}{\partial x^{n}} u(0)
$$

where $\omega=d u(z)$ near $\zeta$.

3. Before proving the theorem we shall first establish a lemma. A subregion $\Omega \subset W$ is called canonical if it is relatively compact, its boundary consists of a finite number of analytic Jordan curves, and each component of $W-\Omega$ is noncompact and bounded by a single contour. For a first order differential $\omega$ let $\int_{\beta} \omega$ indicate the limit as $\Omega \rightarrow W$ of integrals taken along the boundaries of exhausting canonical subregions $\Omega$.

LEммA. Let $p$ be the $(I) L_{1}$ principal function for any singularity. Then $\int_{\beta} p \omega=0$ for all $\omega \in \Gamma_{h}$.

If $\Omega \subset W$ is a canonical subregion with boundary $\partial \Omega$ then $\left|\int_{\partial \Omega} p \omega\right|$ $=\left|\int_{\partial \Omega}\left(p-p_{\Omega}\right) \omega\right|$, where $p_{\Omega}$ is the $(I) L_{1}$ principal function for the surface $\Omega$ which has the same singularity as that of $p$. By Stokes' theorem the right side of this equation is the inner product $\left(d p-d p_{\Omega}, \bar{\omega}^{*}\right)_{\Omega}$ taken over the region $\Omega$. By Schwarz's inequality this inner product is dominated by $\left\|d p-d p_{\Omega}\right\|_{\Omega} \cdot\|\omega\|_{\Omega}$. We therefore obtain $\left|\int_{\beta} p \omega\right| \leqq \lim _{\Omega \rightarrow W}\left\|d p-d p_{\Omega}\right\|_{\Omega} \cdot\|\omega\|_{W}$. The uniform convergence $p_{\Omega} \rightarrow p$ on compact subsets of $W$ implies that $\left\|d p-d_{p_{\Omega}}\right\|_{\Omega} \rightarrow 0$.

For the proof of Theorem 1 we may assume that $\omega$ is real. Let $\Delta$ be a relatively compact neighborhood of $\zeta$ which is mapped by $z$ onto a 
disk $\{|z|<r\}$ and let $\partial \Delta=\alpha$. In order to simplify the notation we shall temporarily denote the functions $p_{I s}$ and $p_{I t}$ by $p$ and $q$ respectively. In $\Delta$ let $\omega=d u(z)$ and $p=\operatorname{Re} z^{-n}+v(z)$ where $u$ and $v$ are harmonic in $\{|z|<r\}$. We then obtain

$$
\begin{aligned}
\left(\omega, d p+d q^{*}\right) & =\iint_{W} \omega\left(d p^{*}-d q\right) \\
& =\iint_{W-\Delta} \omega d p^{*}-\omega d q+\iint_{\Delta} \omega\left(d p^{*}-d q\right) .
\end{aligned}
$$

The integral over $W-\Delta$ is the limit as $\Omega \rightarrow W$ of integrals over $\Omega-\Delta$. We may apply Stokes' theorem to these integrals and, after simplification, we find

$$
\left(\omega, d p+d q^{*}\right)=\int_{\beta}\left(p \omega^{*}+q \omega\right)-\int_{\alpha}\left(p \omega^{*}+p^{*} \omega\right)
$$

The integral along $\beta$ vanishes by the lemma. Hence

$$
\begin{aligned}
\left(\omega, d p+d q^{*}\right) & =-\int_{|z|=r}\left(\operatorname{Re} z^{-n}+v\right) d u^{*}+\left(\operatorname{Im} z^{-n}+v^{*}\right) d u \\
& =\operatorname{Re} \int\left(u^{*}-i u\right) d\left(z^{-n}\right)-\int v d u^{*}-u d v^{*} \\
& =\operatorname{Re} n i \int \frac{u+i u^{*}}{z^{n+1}} d z \\
& =\frac{2 \pi}{(n-1) !} \frac{\partial^{n}}{\partial x^{n}} u(0) .
\end{aligned}
$$

4. We now seek an expression for the reproducing differential for periods. Let $\Delta$ be a parametric disk on $W$ and $\gamma$ a 1 -simplex contained in $\Delta$. In terms of the parameter $z$ which maps $\Delta$ onto the unit disk we define a singularity function $\sigma=\log \left|\left(z-\zeta_{2}\right) /\left(z-\zeta_{1}\right)\right|$, where $\partial \gamma=\zeta_{2}-\zeta_{1}$. We set $\sigma \equiv 0$ outside of a compact set. The corresponding $(P) L_{1}$ principal function is $p_{P \sigma}$. Let $\tau$ be the singularity function $\tau=\arg \left(z-\zeta_{2}\right) /\left(z-\zeta_{1}\right)$ in $\Delta-\gamma$ and $\tau \equiv 0$ near the ideal boundary of $W$. On the surface $W-\gamma$ we choose the normal operator which is composed of $(P) L_{1}$ for a boundary neighborhood of $W$ and of the Dirichlet operator for $\Delta-\gamma$. This Dirichlet operator maps a continuous function on $\partial \Delta$ into the restriction to $\Delta-\gamma$ of the harmonic function in $\Delta$ with these boundary values. The direct sum of these operators yields a function $p_{P \tau}$ harmonic on $W-\gamma$. The differential $d p_{P \tau}$ can be ex- 
tended harmonically to all of $W-\left\{\zeta_{1}, \zeta_{2}\right\}$. We shall continue to denote the extension by $d p_{P \tau}$, even though it is not exact. If $\gamma$ is an arbitrary 1-chain it is homologous to a finite sum $\sum n_{i} \gamma_{i}$ where each $\gamma_{i}$ is a 1 -simplex contained in a parametric disk and each $n_{i}$ is an integer. We extend the definitions of $d p_{P_{r}}$ and $d p_{P_{\tau}}$ to arbitrary $\gamma$ by letting $d p_{P_{\sigma}}=\sum n_{i} d p_{P_{\sigma_{i}}}$ and similarly for $d p_{P_{\tau}}$. That these differentials are well defined will follow from Theorem 2 .

THEOREM 2. The differential $d p_{I_{\sigma}}+d p_{I_{\tau}}^{*}$ reproduces for $\Gamma_{h}$. Specifically, if $\sigma$ and $\tau$ correspond to a 1-chain $\gamma$ then

$$
\left(\omega, d p_{I \sigma}+d p_{I \tau}^{*}\right)=2 \pi \int_{\gamma} \omega
$$

for all $\omega \in \Gamma_{l}$.

Because of the linearity it suffices to prove Theorem 2 for the case that $\gamma$ is a 1 -simplex contained in a parametric disk $\Delta:\{|z|<1\}$ and $\partial \gamma=\zeta_{2}-\zeta_{1}$. We shall shorten the notation and write $p$ and $q$ for $p_{I \sigma}$ and $p_{I \tau}$ respectively. As in the proof of Theorem 1 it can be shown that

$$
\left(\omega, d p+d q^{*}\right)=-\int_{\alpha}\left(p \omega^{*}+p^{*} \omega\right)
$$

where $\partial \Delta=\alpha$. In $\Delta$ let $\omega=d u$ and $p=\log \left|\left(z-\zeta_{2}\right) /\left(z-\zeta_{1}\right)\right|+v$, where $u$ and $v$ are harmonic. Let $\alpha_{1}$ and $\alpha_{2}$ be disjoint circles in $\Delta$ with centers at $z\left(\zeta_{1}\right)$ and $z\left(\zeta_{2}\right)$ respectively. After applying Green's formula one obtains

$$
\left(\omega, d p+d q^{*}\right)=\int_{\alpha_{2}} u d \log \left|z-\zeta_{2}\right|^{*}-\int_{\alpha_{1}} u d \log \left|z-\zeta_{1}\right|^{*} .
$$

By the mean value formula the last two integrals reduce to $2 \pi\left(u\left(\zeta_{2}\right)-u\left(\zeta_{1}\right)\right)$. We have shown that $\left(\omega, d p+d q^{*}\right)=2 \pi \int_{\zeta_{1}}^{\zeta_{2}} d u=2 \pi \int_{\alpha} \omega$. This completes the proof of Theorem 2 .

5. For a regular partition $P$ of the ideal boundary of $W$ the spaces $(P) \Gamma_{h m}$ and $(P) \Gamma_{h s e}$ were introduced in [3], and a proof of the decomposition $\Gamma_{h}=(P) \Gamma_{h m} \dot{+}(P) \Gamma_{h s e}^{*}$ was sketched. It follows that $\Gamma_{h}=\Gamma_{h e}^{*}$ $\dot{+}(P) \Gamma_{h m} \dot{+}(P) \Gamma_{h s e}^{*} \cap \Gamma_{h 0}$ is an orthogonal direct sum. Consider the identity

(1) $-\left(d p_{I s}+d p_{I t}^{*}\right)=\left(d p_{0 t}-d p_{I t}\right)^{*}+\left(d p_{P_{s}}-d p_{I s}\right)-\left(d p_{0 t}^{*}+d p_{P_{s}}\right)$,

where the zero subscript refers to the $L_{0}$ principal function. The first term on the right side of (1) is evidently in $\Gamma_{n e}^{*}$. The second term is 
the limit as $\Omega \rightarrow W$ of $d p_{P_{s} \Omega}-d p_{I_{8} \Omega}$, where the subscript $\Omega$ indicates a principal function for the subsurface $\Omega$. These approximating differentials belong to the space $(P) \Gamma_{h m}(\Omega)$ of harmonic differentials $d u$ on $\Omega$ with $u$ constant on each set of contours of the boundary of $\Omega$ which belong to the same part in the induced partition. We may conclude that the limit differential is in $(P) \Gamma_{h m}$. The last term on the right side of (1) is a limit of elements from $\Gamma_{h 0}(\Omega)$. By Theorem V.14C of [3], we see that it is in $\Gamma_{h 0}$. Its conjugate has vanishing periods along any cycle which is dividing relative to the partition $P$. Hence it is in $(P) \Gamma_{h s e}^{*} \cap \Gamma_{h 0}$. Thus (1) represents the reproducing kernel for $\Gamma_{h}$, except for a multiplicative constant, as the sum of the kernels for $\Gamma_{\text {he }}^{*}$, $(P) \Gamma_{h m}$, and $(P) \Gamma_{h s e}^{*} \cap \Gamma_{h 0}$. The reasoning is also valid for the kernels corresponding to a 1 -chain $\gamma$. This proves the following two corollaries.

Corollary 1. The differentials

$$
\frac{(n-1) !}{2 \pi}\left(d p_{P_{s}}-d p_{I_{s}}\right)
$$

and

$$
\frac{1}{2 \pi}\left(d p_{I \sigma}-d p_{P \sigma}\right)
$$

are the reproducing kernels for the space $(P) \Gamma_{h m}$.

Corollary 2. The differentials

$$
\frac{-(n-1) !}{2 \pi}\left(d p_{P_{s}}+d p_{0 t}^{*}\right)
$$

and

$$
\frac{1}{2 \pi}\left(d p_{P \sigma}+d p_{0 \tau}^{*}\right)
$$

are the reproducing kernels for $(P) \Gamma_{h s e}^{*} \cap \Gamma_{h 0}$.

Since $(I) \Gamma_{h s e}^{*} \cap \Gamma_{h 0}=\Gamma_{h 0}$, Corollary 2 contains the following special case.

Corollary 3. The differentials

$$
\frac{-(n-1) !}{2 \pi}\left(d p_{I_{\bullet}}+d p_{0 t}^{*}\right)
$$


and

$$
\frac{1}{2 \pi}\left(d p_{I \sigma}+d p_{0 \tau}^{*}\right)
$$

are the reproducing kernels for $\Gamma_{h 0}$.

REMARKS. In order to indicate explicitly the relation between the differentials in (1) and the classical domain functions we denote by $N(z, \zeta)$ and $G(z, \zeta)$ the Neumanns' and Green's functions for $W$. In case $W$ is parabolic let $G(z, \zeta)=G\left(z, z_{0} ; \zeta, \zeta_{0}\right)$ be the fundamental potential [4]. In terms of the complex notation

$$
d^{\prime}=\frac{\partial}{\partial z} d z, \quad d^{\prime \prime}=\frac{\partial}{\partial \bar{z}} d \bar{z}, \quad d^{c}=i\left(d^{\prime \prime}-d^{\prime}\right)
$$

(so that $d^{c} f=d f^{*}$ ), we have

$$
\begin{aligned}
d p_{I s}+d p_{I t}^{*} & =\frac{4}{(n-1) !} \operatorname{Re} d^{\prime \prime} \frac{\partial^{n} G}{\partial \zeta^{n}}, \\
\left(d p_{0 t}-d p_{I t}\right)^{*} & =\frac{2}{(n-1) !} \operatorname{Im} d^{c} \frac{\partial^{n}}{\partial \zeta^{n}}(N-G), \\
d p_{I \sigma}+d p_{I \tau}^{*} & =4 \operatorname{Re} \int_{\zeta \in \gamma} d_{z}^{\prime \prime} d_{\zeta}^{\prime} G .
\end{aligned}
$$

Theorems 1 and 2 can also be derived from the fact that $d^{\prime \prime}(\partial G / \partial \zeta)$ reproduces for $\Gamma_{a}$. If $W$ is the interior of a compact bordered surface then by classical theory there is a potential $G_{P}\left(z, z_{0} ; \zeta, \zeta_{0}\right)$ which as a function of $z$ is constant and has zero flux along each $P$-boundary component. In this case we have

$$
\begin{aligned}
& d p_{P_{s}}-d p_{I_{s}}=\frac{2}{(n-1) !} \operatorname{Re} d \frac{\partial^{n}}{\partial \zeta^{n}}\left(G_{P}-G\right), \\
& d p_{0 t}^{*}+d p_{P_{s}}=\frac{2}{(n-1) !} \operatorname{Re}\left(d G_{P}-i d^{c} N\right)
\end{aligned}
$$

6. The space $\Gamma_{h}$ has the orthogonal decomposition

$$
\Gamma_{h}=(P) \Gamma_{h m}+\Gamma_{h 0}^{*} \dot{+}(P) \Gamma_{h s e}^{*} \cap \Gamma_{h e .}
$$

Since we are in possession of reproducing kernels for $(P) \Gamma_{h m}$ and $\Gamma_{h 0}^{*}$ (see the proof of Corollary 1) we obtain the next result immediately.

Corollary 4. The differentials

$$
\frac{(n-1) !}{2 \pi}\left(d p_{0 s}-d p_{P s}\right)
$$


and

$$
\frac{1}{2 \pi}\left(d p_{P \sigma}-d p_{0 \sigma}\right)
$$

are the reproducing kernels for $(P) \Gamma_{h s e}^{*} \cap \Gamma_{h e}$.

The reproducing kernel for $\Gamma_{h e}$ may be derived from Corollary 4.

Corollary 5. The differentials

$$
\frac{(n-1) !}{2 \pi}\left(d p_{0 s}-d p_{I_{s}}\right)
$$

and

$$
\frac{1}{2 \pi}\left(d p_{I \sigma}-d p_{0 \sigma}\right)
$$

are the reproducing kernels for $\Gamma_{h e}$.

7. The kernel for $(P) \Gamma_{h s e}$ can be found from the identity $-\left(d p_{I s}+d p_{l t}^{*}\right)=\left(d p_{P t}^{*}-d p_{l t}^{*}\right)-\left(d p_{I s}+d p_{P l}^{*}\right)$ and the orthogonal decomposition $\Gamma_{h}=(P) \Gamma_{h m}^{*}+(P) \Gamma_{h s e}$.

Corollary 6. The differentials

$$
\frac{-(n-1) !}{2 \pi}\left(d p_{I_{s}}+d p_{P t}^{*}\right)
$$

and

$$
\frac{1}{2 \pi}\left(d p_{I \sigma}+d p_{P r}^{*}\right)
$$

are the reproducing kernels for $(P) \Gamma_{\text {hse. }}$

8. From the orthogonal direct sum,

$$
\Gamma_{h}=(P) \Gamma_{h m}+(P) \Gamma_{h m}^{*}+(P) \Gamma_{a s e}+(P) \bar{\Gamma}_{a s e},
$$

we can project the $\Gamma_{h}$-kernels into $(P) \Gamma_{a s e}$.

COROllary 7. The differentials

$$
\frac{-(n-1) !}{4 \pi}\left(d p_{P_{s}}+d p_{P t}^{*}+i\left(d p_{P s}^{*}-d p_{P t}\right)\right)
$$


and

$$
\frac{1}{4 \pi}\left(d p_{P \sigma}+d p_{P \tau}^{*}+i\left(d p_{P \sigma}^{*}-d p_{P \tau}\right)\right)
$$

are the reproducing kernels for $(P) \Gamma_{a s s}$.

The kernels for $\Gamma_{a}$ and $\Gamma_{a s e}$ may be found immediately from Corollary 7.

REMARKs. It is an open question whether results analogous to those above can be obtained for the subspaces $\Gamma_{S}$ and $\Gamma_{a S}$. Investigations in this area might lead to new normal operators.

9. The results of $\$ \$ 1-8$ indicate a connection between principal functions and Ahlfors' generalization of Abel's theorem [2, 3]. Recall that a differential $\omega$ on $W$, harmonic except for harmonic poles, is called distinguished if

(i) $\omega^{*}$ is semiexact outside of some compact subset of $W$,

(ii) there exist differentials $\omega_{h m} \in \Gamma_{h m}$ and $\omega_{e 0} \in \Gamma_{e 0}^{1}$ such that $\omega=\omega_{h m}+\omega_{e 0}$ in a boundary neighborhood of $W$.

Lemma. Let $\gamma$ be a 1-chain on $W$ and $\delta$ be a 1-cycle. Let $d p_{P \sigma}$ and $d p_{P \tau}$ be differentials associated with $\gamma$ (see $\$ 4$ ). Then

$$
\int_{\delta}\left(d p_{P \sigma}+i d p_{P \tau}\right)=2 \pi i(\delta \times \gamma) \text {. }
$$

Proof. Because (2) is linear in $\gamma$ we may assume that $\gamma$ is a simplex contained in a parametric disk $\Delta:\{|z|<1\}$. Since $d p_{P \sigma}+i d p_{P \tau}$ has no periods along cycles in $W-\Delta$ we may even assume $\delta \subset \bar{\Delta}$. Let $\partial \gamma=\zeta_{2}-\zeta_{1}$. Then $d p_{P \sigma}+i d p_{P \tau}=d \log \left(z-\zeta_{2}\right) /\left(z-\zeta_{1}\right)+d u$ in $\Delta$, where $u$ is harmonic. The proof reduces to establishing

$$
\int_{\delta} d \log \frac{z-\zeta_{2}}{z-\zeta_{1}}=2 \pi i(\delta \times \gamma) .
$$

Since $\delta \times \gamma$ is the number of times $\gamma$ crosses $\delta$ from left to right, (3) follows from the argument principle.

With the help of the above lemma it can be seen that to each distinguished differential $\omega$ there corresponds a differential $\lambda(P, \omega)$ with the singularities and periods of $\omega$ and which, in a boundary neighborhood of $W$, is the differential of a function whose real and imaginary parts have $(P) L_{1}$-behavior.

TheOREm 3. Let $\omega$ be a distinguished differential. Then $\lambda(Q, \omega)=\omega$. 
Proof. Because of the uniqueness theorem for distinguished differentials it suffices to prove merely that $\lambda(Q, \omega)$ satisfies conditions (i) and (ii) above. The first condition is obvious.

The differential $\omega_{h m}=\lambda(Q, \omega)-\lambda(I, \omega)$ is in $\Gamma_{h m}$. Let $\Omega$ be a canonical subregion of $W$ such that $\omega$ is regular and exact in $W-\Omega$. Choose another canonical region $\Omega^{\prime} \supset \bar{\Omega}$. By standard techniques one can construct an exact differential $\omega_{e 0}$ in $\Gamma^{1}$ with the property,

$$
\omega_{e 0}=\left\{\begin{array}{c}
0 \text { in } \Omega, \\
\lambda(I, \omega) \text { in } W-\Omega^{\prime} .
\end{array}\right.
$$

That $\omega_{e 0} \in \Gamma_{e 0}$ follows from the fact that an $(I) L_{1}$ principal function for $W$ is the limit, uniformly on compacta, of $(I) L_{1}$ principal functions for bordered exhausting canonical subregions. The equality $\lambda(Q, \omega)=\omega_{h m}+\omega_{e 0}$ is valid in $W-\Omega^{\prime}$ and hence $\lambda(Q, \omega)$ is distinguished.

From the above considerations one sees that a meromorphic function $f$ is quasi-rational if and only if $\log f$ has a single-valued branch outside of a compact subset of $W$, the real and imaginary parts of which are (Q) $L_{1}$ principal functions.

REMARK. There is a natural interpretation for "quasi-rational with respect to a regular partition $P$." The corresponding generalized Abel's theorem is obtained by replacing $\Gamma_{a s e}$ by $(P) \Gamma_{a s s}$.

10. Riemann-Roch type theorems. The classical theorem of Riemann-Roch has been extended to surfaces of class $O_{K D}$ by Royden [4]. By the methods of this paper we obtain a similar theorem for an arbitrary Riemann surface.

Let $D$ be a divisor on a Riemann surface $W$ and let $D=B-A$ where $A$ and $B$ are disjoint integral divisors. Suppose that $A=\sum m_{j} A_{j}$ and $B=\sum n_{k} B_{k}$, where the $A_{j}$ and $B_{k}$ are points of $W$ and the $m_{j}$ and $n_{k}$ are positive integers. For each $j$ and $k$ let $\Delta_{j}$ and $\Delta_{k}^{\prime}$ be parametric disks with centers at $A_{j}$ and $B_{k}$ respectively. We assume that these disks are mutually disjoint. Let $\Delta=\mathrm{U} \Delta_{j}$ and $\Delta^{\prime}=\mathrm{U} \Delta_{k}^{\prime}$.

Let $S=s+i t$ be a meromorphic function in $\Delta^{\prime}$ and a multiple of the divisor $-B$. Define $S \equiv 0$ in a boundary neighborhood of $W$ and form the differential $d F_{S}=d p_{P_{8}}+i d p_{P t}$. Let $B$ denote the complex vector space consisting of all such $d F_{S}$. The dimension of $B$ is equal to the degree of $B$, i.e., $\operatorname{dim} B=\operatorname{deg} B=\sum n_{k}$.

In the decomposition $d F_{S}=\phi_{S}+\bar{\psi}_{S}$, where $\bar{\psi}_{S}=\frac{1}{2}\left(d F_{S}-i d F_{S}^{*}\right)$, the differential $\phi_{S}$ is meromorphic and $\psi_{S}$ is in $(P) \Gamma_{a s e}$. According to Corollary $7, \psi_{S}$ has certain reproducing properties. The following lemma is a generalization of that fact. For a divisor $E$ let $(P) \Gamma_{a s e}[E]$ denote the vector space of meromorphic differentials which are mul- 
tiples of $E$, square integrable near the ideal boundary of $W$, and which have vanishing periods along each $P$-dividing cycle outside of some compact set.

Lemma. Let $\alpha \in(P) \Gamma_{a s e}[-A]$. Then

$$
\left(\alpha, \psi_{S}\right)_{W-\Delta}=i \int_{\partial \Delta^{\prime}} S \alpha+i \int_{\partial \Delta} F_{S} \alpha .
$$

The inner product is to be understood as a Cauchy limit. That is, it is the limit of inner products taken over $W-\Delta-\Delta^{\prime}$ as the radii of the parametric disks of $\Delta^{\prime}$ tend to zero. Note that

$$
\left(\alpha, \psi_{S}\right)_{W-\Delta-\Delta^{\prime}}=\left(d F_{S}, \bar{\alpha}\right)_{W-\Delta-\Delta^{\prime}}=-i \lim _{\Omega \rightarrow W} \int_{\partial\left(\Omega-\Delta-\Delta^{\prime}\right)} F_{S} \alpha .
$$

A modification of the proof of the lemma in $\$ 3$ shows that $\int_{\partial \Omega} F_{S} \alpha \rightarrow 0$ as $\Omega \rightarrow W$. In $\Delta^{\prime}$ we have $F_{S}=S+u$ where $S$ is meromorphic and $u$ is harmonic. When the disks about the $B_{k}$ shrink to points we obtain (4).

We shall make use of the following algebraic facts $[3$, p. 325]. Let $U$ and $V$ be vector spaces over a field $K$. A bilinear mapping $T: U \times V$ $\rightarrow K$ is called a pairing of $U$ and $V$. The left kernel $U_{0}$ is the space $\{u \in U: T(u, V)=0\}$, and the right kernel $V_{0}$ is the space $\{v \in V: T(U, v)=0\}$. If one of the quotient spaces $U / U_{0}$ or $V / V_{0}$ is finite dimensional then there is an isomorphism $U / U_{0} \cong V / V_{0}$.

Consider the pairing $T: B \times(P) \boldsymbol{\Gamma}_{a s e}[-A] \rightarrow \mathbf{C}$ defined by $T\left(d F_{S}, \alpha\right)$ $=\int_{\partial \Delta^{\prime}} S \alpha$.

Suppose $d F_{S}$ is in the left kernel of this pairing. From (4) we have $\left(\alpha, \psi_{S}\right)_{W-\Delta}=i \int_{\partial \Delta} F_{S} \alpha$ for all $\alpha \in(P) \Gamma_{a s o}[-A]$. We may replace $\alpha$ by $\psi_{S}$ and conclude that $\left\|\psi_{S}\right\|_{W}=0$. Hence $\psi_{S}=0$ and $d F_{S}$ is meromorphic. We also find that

$$
\int_{\partial \Delta} F_{S} \alpha=0
$$

For appropriate choices of $\alpha$ we conclude from (5) that the additive constant in $F_{S}$ can be chosen so that $F_{S}$ is a multiple of the divisor $-D$. Conversely, if $d F_{S}$ is meromorphic then $\psi_{S}=0$ and $T\left(d F_{S}, \alpha\right)$ $=\int_{\partial \Delta^{\prime}} S \alpha=-\int_{\partial \Delta} F_{S} \alpha$. The differential $\alpha$ has a pole of order at most $m_{j}$ at $A_{j}$, and $F_{S}^{(k)}\left(A_{j}\right)=0\left(k=1, \cdots, m_{j}-1\right)$ if $F_{S}$ is a multiple of $A$. Hence $\int_{\partial \Delta} F_{S} \alpha=0$ by Cauchy's integral formula. Thus $d F_{S}$ is in the left kernel $\wp_{0}$ if and only if the function $F_{S}$ is meromorphic and can be normalized so as to be a multiple of $-D$.

A differential $\alpha \in(P) \Gamma_{a s e}[-A]$ is in the right kernel if and only if 
$\int_{\partial \Delta^{\prime}} S \alpha=0$ for all $S$ which are multiples of $-B$. Convenient choices for $S$ show that $\alpha$ must be a multiple of $B$, i.e., $\alpha \in(P) \Gamma_{a s e}[D]$.

Since $B$ is finite dimensional we have

$$
B / B_{0} \cong(P) \Gamma_{a s e}[-A] /(P) \Gamma_{a s e}[D],
$$

or

$$
\operatorname{dim} B_{0}=\operatorname{dim} B-\operatorname{dim}(P) \Gamma_{a s e}[-A] /(P) \Gamma_{a s e}[D] .
$$

Let $(P) M$ be the complex vector space of meromorphic functions on $W$ whose real and imaginary parts have $(P) L_{1}$ behavior near the ideal boundary. Denote by $(P) M[E]$, where $E$ is a divisor, the subspace of $(P) M$ consisting of functions which are multiples of $E$. The homomorphism $d:(P) M[-D] \rightarrow \bigotimes_{0}: F_{S} \rightarrow d F_{S}$ is surjective. The kernel is $\mathbf{C}$ if $\operatorname{deg} A=0$, and it is zero if $\operatorname{deg} A \neq 0$. From (6) we obtain

$$
\begin{aligned}
\operatorname{dim}(P) M[-D]= & \operatorname{deg} B+1-\min (1, \operatorname{deg} A) \\
& -\operatorname{dim}(P) \Gamma_{a s e}[-A] /(P) \Gamma_{a_{s e}}[D] .
\end{aligned}
$$

Theorem 4. Let $A$ and $B$ be disjoint integral divisors on a Riemann surface $W$ and let $D=B-A$. Then (7) is valid for any regular partition $P$ of the ideal boundary of $W$.

\section{BIBLIOGRAPHY}

1. L. V. Ahlfors, The method of orthogonal decomposition for differentials on open Riemann surfaces, Ann. Acad. Sci. Fenn. Ser. A. I. No. 249/7 (1958).

2. - Abel's theorem for open Riemann surfaces, Seminars on analytic functions. II, 7-19. Institute for Advanced Study, Princeton, N. J., 1958.

3. L. V. Ahlfors and L. Sario, Riemann surfaces, Princeton Univ. Press, Princeton, N. J., 1960.

4. H. L. Royden, The Riemann-Roch theorem, Comment. Math. Helv. 34 (1960), $52-66$.

5. L. Sario, A linear operator method on arbitrary Riemann surfaces, Trans. Amer. Math. Soc. 72 (1952), 281-295.

6. G. Weill, Reproducing kernels and orthogonal kernels for analytic differentials on Riemann surfaces, Pacific J. Math. (to appear).

HARVARD UNIVERSITY 